\title{
Morbilidade e Mortalidade da Pneumonia Adquirida na Comunidade no Adulto, em Portugal
}

\author{
Morbidity and Mortality of Community-Acquired Pneumonia in Adults, \\ in Portugal
}

Filipe FROES ${ }^{1,2,3}$

Acta Med Port 2013 Nov-Dec;26(6):644-645

Palavras-chave: Infecções Comunitárias Adquiridas; Hospitalização; Pneumonia/mortalidade; Portugal.

Keywords: Community-Acquired Infections; Epidemiologic Factors; Hospitalization; Pneumonia/mortality; Portugal.

A pneumonia adquirida na comunidade (PAC) é uma causa importante de morbilidade, mortalidade e consumo de recursos de saúde na população adulta. A incidência aumenta com a idade e é maior nos homens do que nas mulheres. ${ }^{1}$

Na última década, estudos em diferentes países ${ }^{2,3}$ documentaram um aumento do número de internamentos hospitalares por PAC. O aumento da esperança média de vida e, consequentemente, da população idosa, a par da crescente prevalência das doenças crónicas não-transmissíveis são alguns dos principais fatores que explicam esta situação.

O aumento dos internamentos por PAC também se verificou em Portugal. ${ }^{4}$ Este aumento só foi possível documentar em Portugal continental por ausência de informação disponível relativa às Regiões Autónomas dos Açores e da Madeira. Assim, no período de 2000 a 2009, a pneumonia adquirida na comunidade foi uma das principais causas de internamento hospitalar no nosso país, representando $3,7 \%$ do total de episódios de internamento hospitalar de adultos por todas as causas em instituições do Serviço $\mathrm{Na}$ cional de Saúde. ${ }^{4}$ Este valor aumentou com a idade, representando $7,0 \%$ nos indivíduos com idade $\geq 65$ anos. ${ }^{4}$ Neste período, foram internados mais homens do que mulheres, $56 \%$ e $44 \%$ respetivamente, e a média de idades dos doentes internados foi de 73 anos. ${ }^{4}$ De destacar que $10 \%$ dos doentes internados tinham idade inferior a 50 anos e $23 \%$ menos de 65 anos. ${ }^{4}$

Ao longo dos 10 anos avaliados constatou-se que o peso dos internamentos por PAC apresentou uma tendência crescente, documentada na evolução deste valor entre os períodos de 2000-2004 a 2005-2009. Verificou-se um aumento da média da percentagem dos internamentos de $3,3 \%$ para $4,2 \%$, o que corresponde a um incremento de $27,3 \%$ de $2000-2004$ para $2005-2009 .{ }^{4}$ Em termos populacionais, o número de internamentos correspondeu a 3,61 internamentos por 1000 habitantes/ $\mathrm{ano}^{4}$ um valor igualmente superior ao documentado em Portugal continental, no período de 1998 a 2000, de 2,66 internamentos por

\section{0 habitantes. $^{5}$}

Nos países mais desenvolvidos, a PAC representa a primeira causa de morte por doença infecciosa ${ }^{6} \mathrm{e}$, de acordo com os últimos dados disponíveis nos Estados Unidos da América (EUA), foi a nona causa de morte em 2010. ${ }^{7}$ Neste país, num estudo realizado em 1996 e envolvendo mais de 33000 doentes, a mortalidade associada à PAC foi avaliada em $13,6 \%$ nos doentes com internamento hospitalar, aumentando para $36,5 \%$ nos doentes admitidos em Unidades de Cuidados Intensivos (UCl). ${ }^{8}$ Num estudo posterior realizado nos EUA de 1987 a 2005 e envolvendo mais de dois milhões de doentes com idade $\geq 65$ anos, a mortalidade aos 30 dias ajustada para a idade e género diminuiu de 13,5 para $9,7 \% .^{9}$

Nos países europeus, a mortalidade atribuível à PAC apresenta grandes variações. Numa revisão publicada em 2011 por Welte et al ${ }^{1}$ com mais de três dezenas de estudos realizados em nove países europeus, verificou-se uma relação significativa entre o risco de mortalidade intra-hospitalar e o aumento de idade, com taxas de letalidade intra-hospitalar que variaram entre os 1,0 e os $43,0 \%$, de acordo com as características das populações avaliadas, nomeadamente a idade (e.g., $\geq 65$ anos), presença de comorbilidades, gravidade (e.g., envolvimento multilobar, choque séptico, necessidade de admissão em UCl), estado imunológico, resposta terapêutica e sensibilidade aos antimicrobianos.

Em Portugal, no triénio de 1998 a 2000, a taxa de letalidade intra-hospitalar dos adultos internados com o diagnóstico principal de PAC foi de $17,3 \% .{ }^{5}$ À semelhança dos internamentos hospitalares, a letalidade intra-hospitalar aumentou para $20,4 \%$ no período de 2000 a $2009 . .^{10}$ Contudo, dada a crescente hospitalização da morte é de considerar a possibilidade deste valor poder estar sobrevalorizado pela inclusão de episódios de pneumonia de fim de vida.

A letalidade variou de acordo com a idade, com valores de $5,0 \%$ nos adultos com idade $<50$ anos e de $22,1 \%$ e $24,1 \%$ nos indivíduos com idades $\geq 50$ e $\geq 65$ anos, respetivamente. ${ }^{10} \mathrm{~A}$ PAC foi responsável por óbitos em todos os

\footnotetext{
1. Consultor de Pneumologia. Direção-Geral da Saúde. Lisboa. Portugal.

2. Departamento de Pneumologia. Faculdade de Medicina de Lisboa. Lisboa. Portugal.

3. Serviço de Pneumologia. Hospital Santa Maria. Centro Hospitalar Lisboa Norte. Lisboa. Portugal.

Recebido: 28 de Agosto de 2013 - Aceite: 30 de Agosto de 2013 | Copyright @ Ordem dos Médicos 2013
} 
grupos etários mesmo em indivíduos jovens previamente saudáveis, verificando-se o valor mais baixo de letalidade de $2,7 \%$ no grupo etário dos 25 aos 29 anos. ${ }^{10}$ Em 3,4\% dos internamentos ocorreu ventilação mecânica invasiva com uma mortalidade média nestes doentes de 44,3\%. ${ }^{10}$

Em conclusão, em Portugal continental verificou-se um aumento significativo dos internamentos hospitalares por PAC, que se traduz num acréscimo do impacto desta doença ao nível da saúde da população e na utilização de recursos do país. Em média, no período de 2000 a 2009, foram internados diariamente 81 adultos com pneumonia

\section{REFERÊNCIAS}

1. Welte T, Torres A, Nathwani D. Clinical and economic burden of community-acquired pneumonia among adults in Europe. Thorax. 2012;67:719.

2. Fry AM, Shay DK, Holman RC, Curns AT, Anderson LJ. Trends in hospitalizations for pneumonia among persons aged 65 years or older in the United States, 1988-2002. JAMA. 2005;294:2712-9.

3. Trotter CL, Stuart JM, George R, Miller E. Increasing hospital admissions for pneumonia, England. Emerging Infect Dis. 2008;14:727-33.

4. Froes F, Diniz A, Mesquita M, Serrado M, Nunes B. Hospital admissions of adults with community-acquired pneumonia in Portugal between 2000 and 2009. Eur Respir J. 2013;41:1141-6.

5. Froes F. Pneumonia da comunidade no adulto em Portugal Continental - incidência e mortalidade dos internamentos hospitalares nos anos de 1998 a 2000. Rev Port Pneumol. 2003;9:187-94. da comunidade dos quais 16 faleceram no decurso desse internamento.

\section{CONFLITO DE INTERESSES}

O autor declara não ter nenhum conflito de interesses relativamente ao presente artigo.

\section{FONTES DE FINANCIAMENTO}

Não existiram fontes externas de financiamento para a realização deste artigo.

6. Niederman MS, McCombs JS, Unger AN, Kumar A, Popovian R. The cost of treating community-acquired pneumonia. Clin Ther. 1998;20:820-37.

7. Murphy SL, Xiu JQ, Kochanek KD. Deaths: Preliminary Data for 2010 . In: National vital statistics reports, editor. Hyattsville: National Center for Health Statistics; 2012.

8. Fine MJ, Smith MA, Carsen CA, Mutha SS, Sankey SS, Weissfeld LA, et al. Prognosis and outcomes of patients with community-acquired pneumonia: A meta-analysis. JAMA. 1996;275:134-41.

9. Ruhnke GW, Coca-Perraillon M, Kitch BT, Cutler DM. Marked reduction in 30-day mortality among elderly patients with community-acquired pneumonia. Am J Med. 2011;124:171.

10. Froes F, Diniz A, Mesquita M, Serrado M, Nunes B. Hospital mortality for CAP in mainland Portugal from 2000 to 2009. Eur Respir J. 2013;41:1141-6. 


\section{Morbilidade e Mortalidade da Pneumonia Adquirida na Comunidade no Adulto, em Portugal \\ Acta Med Port 2013:26:644-645}

Publicado pela Acta Médica Portuguesa, a Revista Científica da Ordem dos Médicos

Av. Almirante Gago Coutinho, 151

1749-084 Lisboa, Portugal.

Tel: +351218428215

E-mail: submissao@actamedicaportuguesa.com

www.actamedicaportuguesa.com

ISSN:0870-399X | e-ISSN: 1646-0758

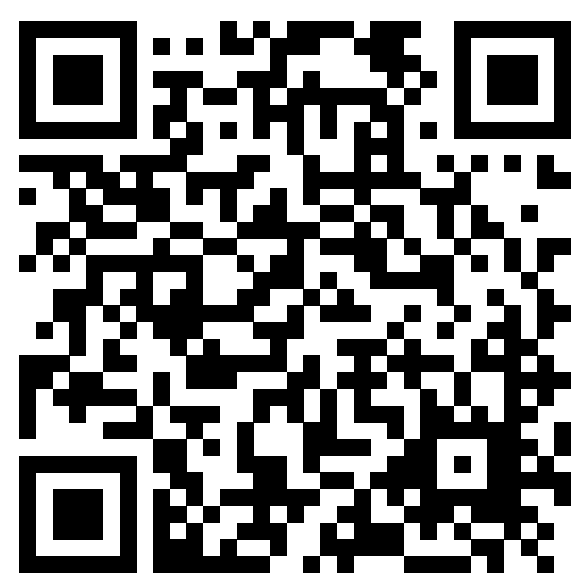

